Pacific

Journal of

Mathematics

A PIERI RULE FOR HERMITIAN SYMMETRIC PAIRS II Thomas J. EnRight and Nolan R. Wallach 


\title{
A PIERI RULE FOR HERMITIAN SYMMETRIC PAIRS II
}

\author{
Thomas J. EnRight and Nolan R. Wallach
}

\begin{abstract}
Let $X$ be an irreducible Hermitian symmetric space of noncompact type and rank $r$. Let $p \in X$ and let $K$ be the isotropy group of $p$ in the group of biholomorphic transformations. Let $S$ denote the symmetric algebra in the holomorphic tangent space to $X$ at $p$. Then $S$ is multiplicity free as a representation of $K$ and the irreducible constituents are parametrized by $r$-tuples, $\left(m_{1}, \ldots, m_{r}\right)$ with $m_{1} \geq \cdots \geq m_{r} \geq 0$. That is, the same parameters as the irreducible polynomial representations of $G L(r)$. Let $S\left[m_{1}, \ldots, m_{r}\right]$ be the corresponding isotypic component. In this article we show that the product in $S, S\left[m_{1}, \ldots, m_{r}\right] S[k, 0,0, \ldots, 0]$ is a direct sum of constituents following precisely the classical Pieri rule.
\end{abstract}

\section{Introduction}

In the classical theory of representations of $G L(r, \mathbb{C})$ the irreducible polynomial representations are parametized by $r$-tuples $\left(m_{1}, \ldots, m_{r}\right)$ of integers with $m_{1} \geq m_{2} \geq \cdots \geq m_{r} \geq 0$. Denoting the corresponding irreducible representation by $F^{m_{1}, \ldots, m_{n}}$ then the Pieri rule $[\mathbf{F}]$ or $[\mathbf{M}]$ says that the tensor product $F^{m_{1}, \ldots, m_{r}} \otimes F^{k, 0, \ldots, 0}$ is a direct sum of irreducible representations with parameters $\left(m_{1}+a_{1}, \ldots, m_{r}+a_{r}\right)$ with $a_{1}+\cdots+a_{r}=k$ and $m_{1}+a_{1} \geq m_{1} \geq m_{2}+a_{2} \geq m_{2} \geq \cdots \geq m_{r}+a_{r} \geq m_{r}$, each occuring with multiplicity one. In this article we will show that there is a completely analogous formula for every irreducible Hermitian symmetric space. We will now describe the result.

Let $X$ be an irreducible Hermitian symmetric space of noncompact type. This means that $X$ is a complex manifold such that, $G$, the group of biholomomrphic transformations of $X$ is simple, noncompact and acts transitively and if $K$ is the stability group of a point $p \in X$ then $K$ is a maximal compact subgroup. Thus we may think of $X=G / K$. We note that the general theory implies that Lie $(K)$ has a one dimensional center, $\mathfrak{Z}$. We set $\mathfrak{g}=\operatorname{Lie}(G) \bigotimes \mathbb{C}$ and $\mathfrak{k}=\operatorname{Lie}(K) \bigotimes \mathbb{C} \subset \mathfrak{g}$. Then $\mathfrak{Z}=\mathbb{R} i H$ and $a d H$ has eigenvalues $0,1,-1$ with eigenspace for 0 being $\mathfrak{k}$ and the eigenspace for 1 identified with the holomorphic tangent space. We will use the standard notation $\mathfrak{p}^{ \pm}=\{X \in \mathfrak{g} \mid[H, X]= \pm X\}$. Thus the holomorphic tangent space is identified with $\mathfrak{p}^{+}$. Using the Killing form of $\mathfrak{g}$ we can identify $\mathfrak{p}^{-}$with $\left(\mathfrak{p}^{+}\right)^{*}$. 
Thus the ring of polynomials on the holomorphic tangent space is identified with $S\left(\mathfrak{p}^{-}\right)$. It is a theorem of Schmid [S] that as a $K$-representation $S\left(\mathfrak{p}^{-}\right)$ is multiplicity free and if $r$ is the rank of $X$ (the dimension of a maximal abelian subalgebra of $\mathfrak{g}$ contained in $\mathfrak{k}^{\perp}$ relative to the Killing form) then the irreducible constituents are labeled (in a natural manner, see the next section for details) by $r$-tuples as in the classical case. We will denote the isotypic component corresponding to $\left(m_{1}, \ldots, m_{r}\right)$ by $S\left(\mathfrak{p}^{-}\right)\left[m_{1}, \ldots, m_{r}\right]$. Then our main result asserts that relative to the multiplication in the algebra $S\left(\mathfrak{p}^{-}\right)$we have $S\left(\mathfrak{p}^{-}\right)\left[m_{1}, \ldots, m_{r}\right] \cdot S\left(\mathfrak{p}^{-}\right)[k, 0, \ldots, 0]$ is the direct sum of the $S\left(\mathfrak{p}^{-}\right)\left[m_{1}+a_{1}, \ldots, m_{r}+a_{r}\right]$ with the $a_{1}, \ldots, a_{r}$ following the classical Pieri rule above. In the last section of this article we show how one can use the classical theory of Littlewood-Richardson combined with the Schmid result to determine that the constituents that can appear in the product are contained in the set described by the Pieri rule in the cases when $G$ is locally isomorphic with $S U(p, q), S O^{*}(2 n)$ and $S p(n, \mathbb{R})$. However, even in these cases the fact that the constituents actually occur is not a clear implication of the classical theory (since the classical theory involves tensor products and not multiplication in a symmetric algebra).

This paper is an extension of some of the results in $[\mathbf{E H W}]$ although it is independent of that article.

\section{K-decomposition of products}

We continue with the Hermitian symmetric setting and the notation of the introduction. Fix a Cartan subalgebra $\mathfrak{h}$ of $\mathfrak{k}$ and a system of positive roots $\Phi^{+}$such that if $\alpha \in \Phi^{+}$then $\alpha(H) \geq 0$. We set $\Phi_{n}^{+}=\{\alpha \in \Phi \mid \alpha(H)=1\}$. Let $\Phi_{c}^{+}=\Phi^{+}-\Phi_{n}^{+}$. Then $\Phi_{c}^{+}$is a system of positive roots for $\mathfrak{k}$ on $\mathfrak{h}$. Let $\gamma_{1}<\gamma_{2}<\cdots<\gamma_{r}$ denote Harish-Chandra's strongly orthogonal roots [HS] which are defined as follows: Let $\gamma_{1}$ denote the unique minimal element of $\Phi_{n}^{+}$. Inductively define $\gamma_{i}$, for $1<i \leq r$, to be the unique minimal element in $\left\{\delta \in \Phi_{n}^{+} \mid\left(\delta, \gamma_{j}\right)=0,1 \leq j<i\right\}$. Schmid [S] has shown that as a $K$-module under the restriction of the adjoint representation $S\left(\mathfrak{p}^{-}\right)$is multiplicity free and the irreducible constituents are exactly the $K$-modules with highest weights of the form

$$
-\left(n_{1} \gamma_{1}+\cdots+n_{r} \gamma_{r}\right), \quad n_{1} \geq n_{2} \geq \cdots \geq n_{r} \geq 0, n_{i} \in \mathbb{Z} .
$$

We write $S\left(\mathfrak{p}^{-}\right)\left[n_{1}, \ldots, n_{r}\right]$ for the corresponding isotypic component. Note that if $d=\sum n_{i}$ then $S\left(\mathfrak{p}^{-}\right)\left[n_{1}, \ldots, n_{r}\right] \subset S^{d}\left(\mathfrak{p}^{-}\right)$, the homogeneous elements of degree $d$. For each positive integer $k$ and multi-index $\underline{n}=$ $\left(n_{1}, n_{2}, \cdots, n_{r}\right)$ with $n_{1} \geq n_{2} \geq \cdots \geq n_{r} \geq 0$, define a set of multi-indices: $I_{k}(\underline{n})=\left\{\underline{m} \mid m_{1} \geq n_{1} \geq m_{2} \geq n_{2} \geq \cdots \geq n_{r-1} \geq m_{r} \geq n_{r} \geq 0\right.$ and $\sum m_{i}=$ $\left.\sum \mathrm{n}_{\mathrm{i}}+\mathrm{k}\right\}$.

The main result of this article is: 
Theorem 1. Let $n_{1} \geq n_{2} \geq \cdots \geq n_{r} \geq 0, n_{i} \in \mathbb{Z}$. Then

$$
S\left(\mathfrak{p}^{-}\right)\left[n_{1}, \ldots, n_{r}\right] \quad S\left(\mathfrak{p}^{-}\right)[k, 0, \ldots, 0]=\bigoplus_{\underline{m} \in I_{k}(\underline{n})} S\left(\mathfrak{p}^{-}\right)[\underline{m}],
$$

with multiplication in $S\left(\mathfrak{p}^{-}\right)$.

We begin with some preliminary definitions and results which will be the basis for a proof by induction on the rank of $\mathfrak{g}$ and the integer $k$. First we recall four well-known results on the strongly orthogonal roots $[\mathrm{Mo}]$ and $[\mathbf{W} 1]$. Let $B$ denote the Killing form of $\mathfrak{g}$. Then $B$ induces a perfect pairing between $\mathfrak{p}^{+}$and $\mathfrak{p}^{-}$. Thus we can look upon the symmetric algebra $S\left(\mathfrak{p}^{-}\right)$ as polynomials on $\mathfrak{p}^{+}$and $S\left(\mathfrak{p}^{+}\right)$as differential operators with constant coefficients on $\mathfrak{p}^{+}$. Let $(.,$.$) denote the dual form to B_{\mid \mathfrak{h}}$.

$$
\begin{aligned}
& \left(\gamma_{i}, \gamma_{i}\right)=\left(\gamma_{j}, \gamma_{j}\right) \text { for all } i, j . \\
& \text { If } \alpha \in \Phi^{+} \text {then }(\alpha, \alpha) \leq\left(\gamma_{1}, \gamma_{1}\right) .
\end{aligned}
$$

Let $\mathfrak{h}^{-}$denote the linear span of the coroots, $\gamma_{i}^{\vee}$, of the $\gamma_{i}$. Then $\operatorname{dim} \mathfrak{h}^{-}=r$ and the $\gamma_{i}^{\vee}$ form an orthogonal basis of $\mathfrak{h}^{-}$.

(2.4) If $\alpha \in \Phi_{n}^{+}$and $\alpha \neq \gamma_{i}$ for any $i$, then $\alpha_{\mid \mathfrak{h}^{-}}$is either of the form $\frac{1}{2}\left(\gamma_{i}+\gamma_{j}\right)$ with $i<j$ or $\frac{1}{2} \gamma_{i}$.

(2.5) If $\alpha \in \Phi_{c}^{+}$then $\alpha_{\mid \mathfrak{h}^{-}}$is either of the form $-\frac{1}{2}\left(\gamma_{i}-\gamma_{j}\right)$ with $i \leq j$ or $-\frac{1}{2} \gamma_{i}$.

Let $\mathfrak{n}_{c}^{+}$denote the sum of the root spaces for the elements of $\Phi_{c}^{+}$. We choose a nonzero element $u_{i}$ in $S\left(\mathfrak{p}^{-}\right)^{\mathfrak{n}_{c}^{+}} \cap S\left(\mathfrak{p}^{-}\right)\left[n_{1}, \ldots, n_{r}\right]$ with $n_{j}=1$ for $j \leq i$ and $n_{j}=0$ for $j>i$. Then one can easily see from Schmid's result that:

(2.6) $S\left(\mathfrak{p}^{-}\right)^{\mathfrak{n}_{c}^{+}}$is the polynomial ring on the algebraically independent elements $u_{1}, \ldots, u_{r}$.

We will now analyze these covariants in further detail. For each $j, 1 \leq$ $j \leq r$, let $\Phi_{0, j}^{+}$denote the set of elements $\alpha$ of $\Phi^{+}$such that $\alpha_{\mid \mathfrak{h}^{-}}$is of the form $\frac{1}{2}\left(\gamma_{p} \pm \gamma_{q}\right)$ with $q \leq p \leq j$. Then $\Phi_{0, j}=\Phi_{0, j}^{+} \cup-\Phi_{0, j}^{+}$is a subrootsystem of $\Phi$. Let $\mathfrak{g}_{0, j}$ denote the subalgebra of $\mathfrak{g}$ generated by the root spaces for the roots in $\Phi_{0, j}$. If $\mathfrak{u}_{j}$ is the sum of all ideals of $\mathfrak{g}_{0, j}$ contained in $\mathfrak{g}_{0, j} \cap \mathfrak{k}$ then $\left(\mathfrak{g}_{0, j} / \mathfrak{u}_{j},\left(\mathfrak{k} \cap \mathfrak{g}_{0, j}\right) / \mathfrak{u}_{j}\right)$ is also an irreducible symmetric pair of Hermitian type $[\mathbf{W} 1]$. Set $\mathfrak{p}_{0, j}^{ \pm}=\mathfrak{g}_{0, j} \cap \mathfrak{p}^{ \pm}$.

Next we introduce a flag of parabolic subalgebras needed for an induction argument. For any root $\alpha$ write $\alpha_{\mid \mathfrak{h}^{-}}=\frac{1}{2} \sum_{1 \leq i \leq r} a_{i} \gamma_{i}$. Then we call $\alpha$ an even (odd) root if the sum $\sum_{1 \leq i \leq r} a_{i}$ is even (odd). We say $\alpha$ has level $j, 1 \leq j \leq r$, if $j$ is the maximal integer with $a_{j} \neq 0$. Otherwise we say $\alpha$ has level 0 . Set $\mathfrak{m}_{j}=\mathfrak{h} \oplus \sum \mathfrak{g}_{\alpha}$ with the sum taken over all even roots of level $\leq j$. Set $\mathfrak{n}_{j}=\mathfrak{n}_{e, j} \oplus \mathfrak{n}_{\text {odd }}$, where $\mathfrak{n}_{e, j}=\sum \mathfrak{g}_{\alpha}$ with the sum taken over 
all even positive roots of level greater than $j$ and $\mathfrak{n}_{\text {odd }}=\sum \mathfrak{g}_{\alpha}$ with the sum taken over all positive odd roots. Finally set $\mathfrak{q}_{j}=\mathfrak{m}_{j} \oplus \mathfrak{n}_{j}$ and let $\mathfrak{b}$ denote the Borel subalgebra with weight spaces corresponding to $\Phi^{+}$.

Lemma 1. For $1 \leq j \leq r, \mathfrak{q}_{j}=\mathfrak{m}_{j} \oplus \mathfrak{n}_{j}$ is a parabolic subalgebra of $\mathfrak{g}$ containing $\mathfrak{b}$ with Levi component $\mathfrak{m}_{j}$ and nilradical $\mathfrak{n}_{j}$. Moreover $\mathfrak{m}_{j}=$ $\mathfrak{h}+\mathfrak{g}_{o, j}$.

Proof. By parity $\mathfrak{m}_{j}$ is a subalgebra of $\mathfrak{g}$. From (2.4) and (2.5), $\mathfrak{n}_{e, j}$ is a subalgebra and infact an ideal in $\mathfrak{m}_{j} \oplus \mathfrak{n}_{e, j}$. Next we chech that $\mathfrak{n}_{\text {odd }}$ is abelian. Split $\mathfrak{n}_{\text {odd }}$ into a direct sum of root spaces for compact and noncompact roots $\mathfrak{n}_{\text {odd }}=\mathfrak{n}_{c, \text { odd }} \oplus \mathfrak{n}_{n \text {,odd }}$. Since $\mathfrak{p}^{+}$is abelian, $\mathfrak{n}_{n, \text { odd }}$ is as well. Suppose $\alpha$ is a positive, odd noncompact root and $\beta$ is positive, odd and compact. But then $\alpha+\beta$ if a root must be positive, even, noncompact and with $(\alpha+\beta)_{\mid \mathfrak{h}^{-}}=\frac{1}{2}\left(\gamma_{i}-\gamma_{l}\right)$. This violates $(2.5)$ and so $\left[n_{n, \text { odd }}, n_{c, \text { odd }}\right]=0$. Similarly assume $\alpha$ and $\beta$ are both positive, odd and compact. Then if a root $\alpha+\beta$ is positive, even, compact and with $(\alpha+\beta)_{\mid \mathfrak{h}^{-}}=\frac{1}{2}\left(-\gamma_{i}-\gamma_{l}\right)$. This violates $(2.5)$ and so $\left[n_{c, \text { odd }}, n_{c, \text { odd }}\right]=0$. Combining these we conclude that $\mathfrak{n}_{\text {odd }}$ is abelian.

Next we show that $\mathfrak{n}_{\text {odd }}$ is an ideal in $\mathfrak{m}_{r} \oplus \mathfrak{n}_{\text {odd }}$. Suppose $\alpha$ is a positive, even root and $\beta$ is positive odd. Then clearly if a root, $\alpha+\beta$ is positive and odd. What remains to show is that if $-\alpha+\beta$ is a root, it also must be positive odd. We proceed in cases. First assume $\alpha$ is noncompact. Then $(-\alpha+\beta)_{\mid \mathfrak{h}^{-}}=\frac{1}{2}\left(-\gamma_{i}-\gamma_{l}\right)+\beta_{\mid \mathfrak{h}}$. So if this is a root we must have cancellation and thus $\beta$ must be noncompact. But then $-\alpha+\beta$ is compact and so the form of $(-\alpha+\beta)_{\mid \mathfrak{h}}$ implies $-\alpha+\beta$ is positive. This proves our claim when $\alpha$ is noncompact. Now suppose $\alpha$ is compact, even and positive. We consider two cases for $\beta$. First assume $\beta$ is positive, odd and compact. If $-\alpha+\beta$ is a root then $(-\alpha+\beta)_{\mid \mathfrak{h}^{-}}=\frac{1}{2}\left(\gamma_{i}-\gamma_{l}\right)+\beta_{\mid \mathfrak{h}}=\frac{1}{2}\left(-\gamma_{l}\right)$. Since $-\alpha+\beta$ is compact this identity implies $-\alpha+\beta$ is positive odd proving the claim in this case. Finally suppose $\beta$ is positive, odd and noncompact. Then if $-\alpha+\beta$ is a root, $(-\alpha+\beta)_{\mid \mathfrak{h}^{-}}=\frac{1}{2}\left(\gamma_{i}-\gamma_{l}\right)+\beta_{\mid \mathfrak{h}}=\frac{1}{2}\left(\gamma_{i}\right)$. Since $-\alpha+\beta$ is noncompact this identity implies $-\alpha+\beta$ is positive odd proving the claim in this last case.

To check that $\mathfrak{n}_{j}$ is an ideal in $\mathfrak{q}_{j}$ we note:

$$
\left[\mathfrak{q}_{j}, \mathfrak{n}_{j}\right] \subset\left[\mathfrak{m}_{j} \oplus \mathfrak{n}_{e, j}, \mathfrak{n}_{e, j}\right] \oplus \mathfrak{n}_{\text {odd }} \subset \mathfrak{n}_{e, j} \oplus \mathfrak{n}_{\text {odd }}=\mathfrak{n}_{j}
$$

This proves $\mathfrak{n}_{j}$ is an ideal in $\mathfrak{q}_{j}$. It follows that the simple roots of $\left(\mathfrak{m}_{j}, \mathfrak{h}\right)$ must also be simple for $(\mathfrak{g}, \mathfrak{h})$. This completes the proof of the lemma.

As an immediate corollary which also follows directly from (2.4) we have:

Suppose $\beta_{i} \in \Phi_{n}^{+}$for $1 \leq i \leq j$ and $\sum_{1 \leq i \leq j} \beta_{i}=\sum_{1 \leq i \leq j} \gamma_{i}$. Then $\beta_{i} \in \Phi_{0, j}$. 
(2.8) Fix $j$ and let $E$ and $F$ be irreducible $\mathfrak{m}_{j}$-modules with highest weights $\xi$ and $\nu$. Let $N(E)$ and $N(F)$ denote the $\mathfrak{g}$-modules obtained by inducing up from $\mathfrak{q}_{j}$ to $\mathfrak{g}$; i.e., $N(E)=U(\mathfrak{g}) \otimes_{U\left(\mathfrak{q}_{j}\right)} E$ and $N(F)=U(\mathfrak{g}) \otimes_{U\left(\mathfrak{q}_{j}\right)} F$. For $\mu \in \mathfrak{h}^{*}$ let a subscript $\mu$ denote the weight space for weight $\mu$. The following weight space identity is an easy consequence of Lemma 1 :

(2.9) Suppose $\mu=\xi+\nu+\sum_{1 \leq i \leq j} a_{i} \gamma_{i}$ with $a_{i} \in \mathbb{Z}$. Then we have an identity of weight spaces:

$$
(N(E) \otimes N(F))_{\mu}=(E \otimes F)_{\mu} .
$$

From [W1] we have:

Lemma 2. For $1 \leq j \leq r, u_{j} \in S\left(\mathfrak{p}_{0, j}^{-}\right)$.

Moreover, for any multi-index $\underline{m}$ with $m_{j+1}=\cdots=m_{r}=0$, then:

$$
\left(S\left(\mathfrak{p}^{-}[\underline{m}]\right)\right)^{\mathfrak{n}_{c}}=\left(S\left(\mathfrak{p}_{0, j}^{-}[\underline{m}]\right)\right)^{\mathfrak{n}_{c} \cap \mathfrak{g}_{0, j}} .
$$

By Lemma 2 the highest weight vectors although not the full $\mathfrak{k}$-modules are contained in $S\left(\mathfrak{p}_{0, r}^{-}\right)$. As a first reduction we claim it is sufficient to prove Theorem 1 when $\mathfrak{g}=\mathfrak{g}_{0, r}$. To this end set $E=S\left(\mathfrak{p}_{0, j}^{-}\right)\left[n_{1}, \ldots, n_{j}\right]$ and $F=S\left(\mathfrak{p}_{0, j}^{-}\right)[k, 0, \ldots, 0]$. If $\mu$ is a highest weight of the form $\mu=\sum_{1 \leq i \leq j} a_{i} \gamma_{i}$ then by (2.9), we obtain:

$$
\begin{aligned}
& \left(S\left(\mathfrak{p}^{-}\right)\left[n_{1}, \ldots, n_{j}, 0, \ldots, 0\right] \quad S\left(\mathfrak{p}^{-}\right)[k, 0, \ldots, 0]\right)_{\mu} \\
& =\left(S\left(\mathfrak{p}_{0, j}^{-}\right)\left[n_{1}, \ldots, n_{j}\right] \quad S\left(\mathfrak{p}_{0, j}^{-}\right)[k, 0, \ldots, 0]\right)_{\mu},
\end{aligned}
$$

where on the right the bracket designates the $\mathfrak{k} \cap \mathfrak{g}_{0, j}$ isotypic subspaces.

From this identity (2.10) with $j=r$ and Lemma 2 we conclude that Theorem 1 holds for $\mathfrak{g}$ if and only if it holds for $\mathfrak{g}_{0, r}$.

From this point forward we assume $\mathfrak{g}=\mathfrak{g}_{0, r}$. This reduction has several simplifying features which we now summarize. By (2.5) for any compact root $\delta, \delta_{\mid \mathfrak{h}^{-}}=\frac{1}{2}\left(\gamma_{i}-\gamma_{l}\right)$. This implies that the $\mathfrak{k}$-module $S\left(\mathfrak{p}^{-}\right)[n, n, \ldots, n]$ is one dimensional. If $\delta$ is positive and compact then for some $i<j, \delta_{\mid \mathfrak{h}^{-}}+\gamma_{r}=$ $\frac{1}{2}\left(-\gamma_{i}+\gamma_{l}\right)+2 \gamma_{r}$ which contradicts $(2.5)$. This proves $\gamma_{r}$ is a maximal root with respect to $\mathfrak{k}$. This same identity shows that the weight spaces $\mathfrak{g}_{\gamma_{r}}$ and $\mathfrak{g}_{-\gamma_{r}}$ are one dimensional $\mathfrak{k} \cap \mathfrak{g}_{0, r-1}$-modules. Since $\mathfrak{p}^{+}$is abelian, $\gamma_{r}$ is the maximal weight in $\Phi^{+}$.

From the one dimensionality of $S\left(\mathfrak{p}^{-}\right)[n, n, \ldots, n]$, we obtain:

$$
S\left(\mathfrak{p}^{-}\right)\left[n_{1}, \ldots, n_{r}\right]=S\left(\mathfrak{p}^{-}\right)\left[n_{1}-n_{r}, \ldots, n_{r-1}-n_{r}, 0\right] u_{r}^{n_{r}} .
$$

Lemma 3. Suppose Theorem 1 holds for $\mathfrak{g}_{0, r-1}, n_{r}=0$ and $\underline{m}$ is a multiindex not in $I_{k}(\underline{n})$ whose $K$-type occurs in the product in the left-hand side of Equation (2.1). Then $m_{r} \geq 1$.

Proof. Let $E=S\left(\mathfrak{p}_{0, r-1}^{-}\right)\left[n_{1}, \ldots, n_{r-1}, 0\right]$ and $F=S\left(\mathfrak{p}_{0, r-1}^{-}\right)[k, 0, \ldots, 0]$ and let $v$ be a highest weight vector of the $K$-type indexed by $\underline{m}$. By hypothesis 
the $\mathfrak{k} \cap \mathfrak{g}_{0, r-1}$-decomposition of $E \cdot F$ shows that $v$ is not in $E \cdot F$. So by (2.10) with $j=r-1$, we have a contradiction unless $m_{r} \geq 1$.

We denote by $x \mapsto \bar{x}$ the conjugation of $\mathfrak{g}$ with respect to $\operatorname{Lie}(G)$. Then $\overline{\mathfrak{p}^{-}}=\mathfrak{p}^{+}$. If $x \in \mathfrak{p}^{+}$then we denote by $\partial(x)$ the derivation of $S\left(\mathfrak{p}^{-}\right)$defined by $\partial(x) y=B(x, y)$ for $y \in \mathfrak{p}^{-}$. We will also denote the extension of $\partial$ to $S\left(\mathfrak{p}^{+}\right)$by $\partial$. In addition we will use the notation $u \mapsto u(0)$ for the augmentation map of $S\left(\mathfrak{p}^{-}\right)$to $\mathbb{C}$ given as the extension to a homomorphism of $y \mapsto 0$ for $y \in \mathfrak{p}^{-}$. We define for $u, v \in S\left(\mathfrak{p}^{-}\right),\langle u, v\rangle=(\partial(\bar{v}) u)(0)$. For any root $\beta$ let $X_{\beta}$ denote an element in the root space $\mathfrak{g}_{\beta}$ normalized so that $\bar{X}_{\beta}=X_{-\beta}$.

The following observation is well-known and easily checked:

(2.12) The Hermitian form $\langle.,$.$\rangle is positive definite and K$-invariant. Furthermore, if $u, v, w \in S\left(\mathfrak{p}^{-}\right)$then $\langle u v, w\rangle=\langle v, \partial(\bar{u}) w\rangle$. If $n_{1} \geq \cdots \geq n_{r} \geq 0$ then we note that $u_{1}^{n_{1}-n_{2}} u_{2}^{n_{2}-n_{3}} \cdots u_{r-1}^{n_{r-1}-n_{r}} u_{r}^{n_{r}}$ is a basis of the highest weight space of $S\left(\mathfrak{p}^{-}\right)\left[n_{1}, \ldots, n_{r}\right]$. Let $D=\partial\left(\overline{u_{r}}\right)$. Then

$$
D: S\left(\mathfrak{p}^{-}\right)\left[n_{1}, \ldots, n_{r}\right] \rightarrow S\left(\mathfrak{p}^{-}\right)\left[n_{1}-1, \ldots, n_{r}-1\right] .
$$

Here if $n_{r}<0$ then we write $S\left(\mathfrak{p}^{-}\right)\left[n_{1}, \ldots, n_{r}\right]=\{0\}$.

The maps $D$ and multiplication by $u_{r}$ are semi-invariant maps and the product $u_{r} D$ is a $K$-invariant map which plays an important role below. The eigenvalues of this operator are given in:

Lemma 4. For each multi-index $\underline{n}$ the operator $u_{r} D$ is diagonalizable and there are nonzero constants $c$ and $C_{2}$ such that $u_{r} D$ restricted to $S\left(\mathfrak{p}^{-}\right)[\underline{n}]$ equals

$$
C_{2} \prod_{i=1}^{r}\left(\frac{r-i}{2} c+n_{i}\right) \cdot I .
$$

Proof. This is the first case of Theorem 3.3 in [W2].

Remark 1. The referee has noted that Lemma 4 is employed only to show that (2.13) is a bijective map for all $n_{r} \geq 1$. An alternative argument can be given since $D$ is the adjoint to multiplication by $u_{r}$.

Lemma 5. Suppose $\underline{n}$ is a multi-index with $n_{r}=0$, set

$$
v=u_{1}^{n_{1}-n_{2}} u_{2}^{n_{2}-n_{3}} \cdots u_{r-1}^{n_{r-1}} .
$$

Then

$$
D\left(v X_{-\gamma_{r}}^{k}\right)=C_{1} u_{1}^{n_{1}-n_{2}} u_{2}^{n_{2}-n_{3}} \cdots u_{r-2}^{n_{r-2}-n_{r-1}} u_{r-1}^{n_{r-1}-1} X_{-\gamma_{r}}^{k-1},
$$

with $C_{1}=0$ if and only if $n_{r-1}=0$.

Moreover $D$ is a $K$-intertwining operator that carries a cyclic vector for the product $S\left(\mathfrak{p}^{-}\right)\left[n_{1}, \ldots, n_{r-1}, 0\right] S\left(\mathfrak{p}^{-}\right)[k, 0, \ldots, 0]$ to a cyclic vector for the product $S\left(\mathfrak{p}^{-}\right)\left[n_{1}-1, \ldots, n_{r-1}-1,0\right] S\left(\mathfrak{p}^{-}\right)[k-1,0, \ldots, 0]$. 
Proof. The identity (2.15) is a consequence of calculations found in [W1] (see the proofs of Lemmas 5.7 and 5.8). Since the identity is so essential in our proof of Theorem 1 we repeat the argument here.

Expanding with respect to noncompact root vectors we choose constants $a_{\beta_{1}, \ldots, \beta_{r}}$ with

$$
\begin{gathered}
u_{r}=\sum a_{\beta_{1}, \ldots, \beta_{r}} X_{-\beta_{1}} \cdots X_{-\beta_{r}} \quad \text { and } \\
u_{r}=u X_{-\gamma_{r}}+w \quad \text { where } \quad w=\sum a_{\beta_{1}, \ldots, \beta_{r}} X_{-\beta_{1}} \cdots X_{-\beta_{r}},
\end{gathered}
$$

and the first sum is over all $\beta_{1} \leq \cdots \leq \beta_{r}$ with $\sum_{1 \leq i \leq r} \beta_{i}=\sum_{1 \leq i \leq r} \gamma_{i}$ and the second sum is over the subset where $\beta_{i} \neq \gamma_{r}$ for all $i$. The identity (2.7) implies that $u \in S\left(\mathfrak{p}_{0, r-1}^{-}\right)$. Moreover from our reduction $\mathfrak{g}=\mathfrak{g}_{0, r}$ we know $u$ is a semi-invariant for $\mathfrak{k} \cap \mathfrak{g}_{0, r-1}$. This implies that $u$ is a scalar multiple of $u_{r-1}$. Computing the action of $D=\partial \bar{u}_{r}$ on $v X_{-\gamma_{r}}^{k}$, we obtain: $D\left(v X_{-\gamma_{r}}^{k}\right)=k \partial \bar{u}(v) X_{-\gamma_{r}}^{k-1}$. Since $u$ is a scalar multiple of the $\mathfrak{k} \cap \mathfrak{g}_{0, r-1}$ semiinvariant $u_{r-1} \in S\left(\mathfrak{p}_{0, r-1}^{-}\right)$, we may apply (2.13) with $r$ replaced with $r-1$ to obtain the identity (2.15).

The highest weight space times the lowest weight space is cyclic for the tensor product of any two irreducible finite dimensional representations. So the second assertion follows from the identity.

Proof of Theorem 1. We will prove the theorem by induction on $r$ and then by induction on $k$. If $r=1$ then by an earlier reduction $\mathfrak{g}=\mathfrak{g}_{0,1} \cong \operatorname{sl}(2)$ and the result is trivial in this case. Now assume the theorem for $r-1 \geq 1$. If $k=0$ there is nothing to prove so assume the theorem for $k-1 \geq 0$. We now prove the result for $k$.

As above we may assume $\mathfrak{g}=\mathfrak{g}_{0, r}$. By identity (2.11) we may assume $n_{r}=0$. If $n_{r-1}=0$ as well we restrict our attention to $\mathfrak{g}_{0, r-1}$. By the induction hypothesis the theorem holds for $\mathfrak{g}_{0, r-1}$ so we obtain by Lemma 2 the inclusion

$$
S\left(\mathfrak{p}^{-}\right)\left[n_{1}, \ldots, n_{r}\right] \quad S\left(\mathfrak{p}^{-}\right)[k, 0, \ldots, 0] \supset \bigoplus_{\underline{m} \in I_{k}(\underline{n})} S\left(\mathfrak{p}^{-}\right)[\underline{m}] .
$$

Now we prove equality here. Suppose not. Then by Lemma 3 , there is an index $\underline{m} \notin I_{k}(\underline{n})$ with $m_{r} \geq 1$ whose $K$-type contains a vector $z$ occurring on the left side. By Lemma $4, z$ is an eigenvector for $u_{r} D$ with nonzero eigenvalue. However by Lemma 5 and especially the cyclicity of $v X_{-\gamma_{r}}^{k}, D$ acts by zero on the left side of (2.1) in Theorem 1, which is a contradiction. This proves the case $n_{r-1}=n_{r}=0$.

Now assume $n_{r-1}>0, n_{r}=0$. Set $\underline{n}^{\prime}=\left(n_{1}-1, n_{2}-1, \cdots, n_{r-2}-\right.$ $\left.1, n_{r-1}-1,0\right)$. By Lemma 5 we obtain:

$$
D\left(S\left(\mathfrak{p}^{-}\right)[\underline{n}] S\left(\mathfrak{p}^{-}\right)[k, 0, \ldots, 0]\right)=S\left(\mathfrak{p}^{-}\right)\left[\underline{n}^{\prime}\right] S\left(\mathfrak{p}^{-}\right)[k-1,0, \ldots, 0] .
$$


Also by the induction hypothesis for $k$,

$$
S\left(\mathfrak{p}^{-}\right)\left[\underline{n}^{\prime}\right] S\left(\mathfrak{p}^{-}\right)[k-1,0, \ldots, 0] \cong \sum_{\underline{m} \in I_{k-1}\left(\underline{n}^{\prime}\right)} S\left(\mathfrak{p}^{-}\right)[\underline{m}] .
$$

Now combining these identities and multiplying by $u_{r}$, we conclude:

$$
\begin{aligned}
& S\left(\mathfrak{p}^{-}\right)[\underline{n}] S\left(\mathfrak{p}^{-}\right)[k, 0, \ldots, 0] \supset u_{r} D\left(S\left(\mathfrak{p}^{-}\right)[\underline{n}] S\left(\mathfrak{p}^{-}\right)[k, 0, \ldots, 0]\right) \\
& =\bigoplus_{\underline{m} \in I_{k}(\underline{n}), m_{r} \geq 1} S\left(\mathfrak{p}^{-}\right)[\underline{m}] .
\end{aligned}
$$

Since $n_{r}=0$ we can restrict to $\mathfrak{g}_{0, r-1}$. From Lemma 2 we have:

$$
\begin{aligned}
& S\left(\mathfrak{p}^{-}\right)[\underline{n}] S\left(\mathfrak{p}^{-}\right)[k, 0, \ldots, 0] \supset \quad \bigoplus \quad S\left(\mathfrak{p}^{-}\right)[\underline{m}] . \\
& \underline{m} \in I_{k}(\underline{n}), m_{r}=0
\end{aligned}
$$

Combining these two inclusions we get:

$$
S\left(\mathfrak{p}^{-}\right)[\underline{n}] \quad S\left(\mathfrak{p}^{-}\right)[k, 0, \ldots, 0] \supset \bigoplus_{\underline{m} \in I_{k}(\underline{n})} S\left(\mathfrak{p}^{-}\right)[\underline{m}] .
$$

We now prove equality. Suppose not and choose $\underline{m}$ with the corresponding $K$-type containing a vector $z$ occurring on the left but not on the right in (2.1) in Theorem 1. Applying Lemma 3 we find $m_{r} \geq 1$. This inequality implies that $u_{r} D z$ is a nonzero multiple of $z$. So $z$ is contained in the $K$-representation generated by $D\left(v X_{-\gamma_{r}}^{k}\right)$. By $(2.19) \underline{m} \in I_{k}(\underline{n})$, a contradiction. This completes the proof of the theorem.

\section{Connections with the classical Pieri rule}

For the classical cases of $S p(n, \mathbb{R}), S O^{*}(2 n)$ and $U(p, q)$, the decomposition of products given by Theorem 1 is related to the Pieri rule for $G l(n)$ for the first and third cases and to the Littlewood-Richardson rule for $G l(n)$ in the case of $S O^{*}(2 n)$. We handle each case separately and keep in place the earlier notation. For these classical cases we use the standard Euclidean coordinates $e_{i}$ as in Bourbaki.

Case $1, S p(n, \mathbb{R})$. Here $\mathfrak{k} \cong u(n)$ and for $1 \leq i \leq n, \gamma_{i}=2 e_{n+1-i}$. Set $E=S\left(\mathfrak{p}^{-}\right)\left[m_{1}, \ldots, m_{n}\right]$ and $F=S\left(\mathfrak{p}^{-}\right)[k, 0, \ldots, 0]$. Let a ${ }^{*}$ denote the dual representation. Then $E^{*}$ and $F^{*}$ have highest weights $\left(2 m_{1}, \ldots, 2 m_{n}\right)$ and $(2 k, 0,0, \ldots, 0)$ respectively. Then applying the Pieri rule, we obtain:

$$
E^{*} \otimes F^{*} \cong \sum E_{\underline{b}},
$$

where the sum is over all $n$-tuples $\underline{b}=\left(b_{1}, b_{2}, \cdots, b_{n}\right)$ with $b_{1} \geq 2 m_{1} \geq$ $b_{2} \geq 2 m_{2} \geq \cdots \geq b_{n} \geq 2 m_{n}$ and $\sum b_{i}-2 m_{i}=2 k$. From Schmid's result combined with this identity we obtain the inclusion in $S\left(\mathfrak{p}^{-}\right)$:

$$
E \cdot F \subset \sum E_{\underline{b}}^{*}
$$


where the sum is over all $n$-tuples $\underline{b}=\left(b_{1}, b_{2}, \ldots, b_{n}\right)$ with even entries, $b_{1} \geq 2 m_{1} \geq b_{2} \geq 2 m_{2} \geq \cdots \geq b_{n} \geq 2 m_{n}$ and $\sum b_{i}-2 m_{i}=2 k$. Applying Theorem 1 in this setting we conclude that the inclusion is an equality. So the multiplicative product is as large as it could be.

Case 2, $S O^{*}(2 n)$. Here $\mathfrak{k} \cong u(n)$ and for $1 \leq i \leq r=\left[\frac{n}{2}\right], \gamma_{i}=e_{n+1-2 i}+$ $e_{n+2-2 i}$. Set $E=S\left(\mathfrak{p}^{-}\right)\left[m_{1}, m_{2}, \ldots, m_{r}\right]$ and $F=S\left(\mathfrak{p}^{-}\right)[k, 0, \ldots, 0]$. Then $E^{*}$ and $F^{*}$ have highest weights $\left(m_{1}, m_{1}, m_{2}, m_{2}, \ldots\right)$ and $(k, k, 0, \ldots, 0)$ respectively. The Littlewood-Richardson rule $[\mathbf{F}]$ or $[\mathbf{M}]$ gives the decomposition of $E^{*} \otimes F^{*}$.

$$
E^{*} \otimes F^{*} \cong \sum m_{\underline{b}} E_{\underline{b}},
$$

where the multiplicity of $E_{\underline{b}}$, denoted $m_{\underline{b}}$ equals the number of ways the diagram $\underline{m}=\left(m_{1}, m_{1}, m_{2}, m_{2}, \ldots\right)$ can be expanded to the diagram $\underline{b}$ by a strict $(k, k, 0, \cdots, 0)$-expansion. Such an expansion is determined by augmenting the diagram $\underline{m}$ by adding $k$ boxes each labeled with a 1 and then adding $k$ boxes each labeled with a 2 so that the following three conditions hold:

(i) The labels in each row of are nondecreasing,

(ii) the labels in each column of are strictly increasing and

(iii) if we read the labels from top right to bottom left at every stage the number 1 must have occured at least as many times as the number 2 .

As an example, suppose $\underline{m}=(3,3,1,1)$ and $\underline{k}=(2,2,0,0)$. Then all the multiplicities are one and the $\mathfrak{k}$-modules which occur are: $(5,5,1,1)(5,4,2,1)$ $(5,3,3,1)(4,4,2,2)(4,3,3,2)(3,3,3,3)$.

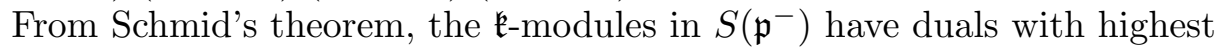
weights whose diagrams have columns of even length. Since $\underline{m}$ has columns of even length, the rules (i), (ii), and (iii) imply that the only placements of $k 1^{\prime} s$ and $k 2^{\prime} s$ which yield a diagram with columns of even length will have the form $\underline{b}=\left(b_{1}, b_{1}, b_{2}, b_{2}, \ldots\right)$ with $\sum b_{i}-m_{i}=k$ and $b_{1} \geq m_{1} \geq b_{2} \geq$ $m_{2} \geq \cdots \geq b_{r} \geq m_{r}$. So here the Littlewood-Richardson rule and Schmid's Theorem combine to give the inclusion:

$$
E^{*} \cdot F^{*} \subset \sum E_{\underline{b}},
$$

where the sum is over all $\underline{b}$ with $\underline{b}=\left(b_{1}, b_{1}, b_{2}, b_{2}, \ldots\right), \sum b_{i}-m_{i}=k$ and $b_{1} \geq m_{1} \geq b_{2} \geq m_{2} \geq \cdots \geq b_{r} \geq m_{r}$. Our Theorem 1 asserts that this inclusion is equality.

Case $3, U(p, q)$. Set $n=p+q$ and assume $p \leq q$. Here $\mathfrak{k} \cong u(p) \oplus u(q)$ and for $1 \leq i \leq p, \gamma_{i}=e_{p+1-i}-e_{p+i}$. Set $E=S\left(\mathfrak{p}^{-}\right)\left[m_{1}, m_{2}, \ldots, m_{p}\right]$ and $F=S\left(\mathfrak{p}^{-}\right)[k, 0, \ldots, 0]$. Since $\mathfrak{k}$ is a product of ideals $\mathfrak{k}=\mathfrak{k}^{(1)} \oplus \mathfrak{k}^{(2)}$ and every irreducible $\mathfrak{k}$-module splits into a tensor product. We write $E \cong E^{(1) *} \otimes$ $E^{(2)}, F \cong F^{(1) *} \otimes F^{(2)}$ where the highest weights of $E^{(1)}, E^{(2)}, F^{(1)}, F^{(2)}$ are 
respectively:

$$
\begin{gathered}
\underline{m}^{(1)}=\left(m_{1}, m_{2}, \ldots, m_{p}\right), \quad m^{(2)}=\left(m_{1}, m_{2}, \ldots, m_{p}, 0, \cdots, 0\right), \\
(k, 0, \ldots, 0),(k, 0, \ldots, 0) .
\end{gathered}
$$

Here the first and third are $p$-tuples and the second and forth are $q$-tuples. Applying the Pieri rule for each factor we obtain:

$$
\left(E^{(1)} \otimes E^{(2)}\right) \otimes\left(F^{(1)} \otimes F^{(2)}\right) \cong \sum E_{\underline{b}}^{(1)} \otimes E_{\underline{c}}^{(2)},
$$

where the sum is over all $p$-tuples $\underline{b}$ and all $q$-tuples $\underline{c}$ which satisfy the conditions:

$$
\begin{aligned}
& \sum_{1 \leq i \leq p} b_{i}-m_{i}=k, \quad b_{1} \geq m_{1} \geq b_{2} \geq m_{2} \geq \cdots \geq b_{p} \geq m_{p}, \quad \text { and } \\
& \sum_{1 \leq i \leq q} c_{i}-m_{i}=k, \quad c_{1} \geq m_{1} \geq c_{2} \geq m_{2} \geq \cdots \geq c_{p} \geq m_{p} \geq c_{p+1} \geq 0,
\end{aligned}
$$

with $0=c_{p+2}=\cdots=c_{q}$.

By Schmid's Theorem the only summands which occur in $S\left(\mathfrak{p}^{-}\right)$are those $E_{\underline{b}}^{(1) *} \otimes E_{\underline{c}}^{(2)}$ for which $b_{i}=c_{i}, 1 \leq i \leq p$ and $c_{i}=0, p+1 \leq i \leq q$. So taking the product in $S\left(\mathfrak{p}^{-}\right)$we obtain the inclusion:

$$
\left(E^{(1) *} \otimes E^{(2)}\right) \cdot\left(F^{(1) *} \otimes F^{(2)}\right) \subset \sum E_{\underline{b}}^{(1) *} \otimes E_{\underline{c}}^{(2)},
$$

where $\sum_{1 \leq i \leq p} b_{i}-m_{i}=k, b_{1} \geq m_{1} \geq b_{2} \geq m_{2} \geq \cdots \geq b_{p} \geq m_{p}, b_{i}=$ $c_{i}, 1 \leq i \leq p$ and $c_{i}=0, p+1 \leq i \leq q$. Finally as before Theorem 1 asserts the inclusion is equality.

\section{References}

[EHW] T.J. Enright, M. Hunziker and N.R. Wallach, A Pieri rule for Hermitian symmetric pairs I, Pacific J. Math., 214 (2004), 23-30.

[F] W. Fulton, Young Tableaux, Cambridge University Press, Cambridge, 1997, MR 1464693 (99f:05119), Zbl 0878.14034.

[HS] Harish-Chandra, Representations of semisimple Lie groups VI, Amer. J. Math., 78 (1956), 564-628, MR 0082056 (18,490d), Zbl 0072.01702.

[M] I.G. Macdonald, Symmetric Functions and Hall Polynomials, Clarendon Press, Oxford, 1979, MR 0553598 (84g:05003), Zbl 0487.20007.

[Mo] C.C. Moore, Compactifications of symmetric spaces. II. The Cartan domains, Amer. J. Math., 86 (1964), 358-378, MR 0161943 (28 \#5147), Zbl 0156.03202.

[S] W. Schmid, Die Randwerte holomorpher Funktionen auf Hermitesch symmetrischen Räumen, Invent. Math., 9 (1969-70), 61-80, MR 0259164 (41 \#3806), Zbl 0156.03202.

[W1] N.R. Wallach, The analytic continuation of the discrete series I and II, Trans. Amer. Math. Soc., 251 (1979), 1-17 and 19-37, MR 0531967 (81a:22009), Zbl 0419.22017, Zbl 0419.22018. 
[W2] Polynomial differential operators associated with Hermitian symmetric spaces, in 'Representation theory of Lie groups and Lie algebras' (FujiKawaguchiko, 1990), 76, 1994, World Sci. Publishing, River Edge, NJ, 1992, 76-94, MR 1190751 (94a:22031).

Received April 11, 2003 and revised November 5, 2003. The research of the second author was supported in part by an NSF summer grant.

Department of Mathematics

University of California, San Diego

LA Jolla, CA 92093

E-mail address: tenright@ucsd.edu

Department of Mathematics

University of California, San Diego

LA Jolla, CA 92093

E-mail address: nwallach@ucsd.edu 